Article

\title{
Feasibility Study of Glucose Concentration Measurement of Aqueous Solution Using Time Domain Reflected Signals
}

\author{
Samira Saeedi ${ }^{1,2}$, Somayyeh Chammani ${ }^{1, *(D)}$ and Georg Fischer ${ }^{2}$ (D) \\ 1 Time-Domain Electromagnetics Laboratory, Faculty of Electrical Engineering, \\ K.N. Toosi University of Technology, Tehran 1631714191, Iran; samirasaeedi@email.kntu.ac.ir \\ 2 Institute for Electronics Engineering, University of Erlangen, 91058 Nuremberg, Germany; \\ georg.fischer@fau.de \\ * Correspondence: chamaani@kntu.ac.ir
}

check for updates

Citation: Saeedi, S.; Chammani, S.; Fischer, G. Feasibility Study of Glucose Concentration Measurement of Aqueous Solution Using Time Domain Reflected Signals. Sensors 2022, 22, 1174. https://doi.org/ $10.3390 / \mathrm{s} 22031174$

Academic Editor: Toshiyo Tamura

Received: 12 November 2021

Accepted: 1 February 2022

Published: 3 February 2022

Publisher's Note: MDPI stays neutral with regard to jurisdictional claims in published maps and institutional affiliations.

Copyright: (C) 2022 by the authors. Licensee MDPI, Basel, Switzerland. This article is an open access article distributed under the terms and conditions of the Creative Commons Attribution (CC BY) license (https:// creativecommons.org/licenses/by/ $4.0 /)$.

\begin{abstract}
Recently, wideband microwave spectroscopy (WBMS) has been applied for material characterization. Blood glucose sensing through microwave spectroscopy is usually done with resonant frequency-domain methods. Time-domain (TD) WBMS is a low-cost and convenient technique that can be used for glucose sensing of the aqueous solution. In this paper, early research for the implementation of a TD dielectric spectroscopy setup for glucose concentration measurement is presented. TD reflected signals from water with different glucose content are calculated using inverse Laplace transform. The proposed setup is a quasi-monostatic setup in which measurements are done with two different devices in the frequency range of 0.1 to $6 \mathrm{GHz}$ to make a comparison between frequency domain (FD) and TD methods. Frequency domain (FD) measurement is performed with VNA and two Vivaldi antennas. Then, TD data is obtained using the transforming option of VNA. Direct TD measurement is operated with a maximum length sequence (m-sequence) transceiver. Measurement and numerical results follow the same trend and show good agreement with each other. A monotonic relation between peaks of TD signals and the corresponding glucose concentration is achieved. The variation of the height of the reflected signal's peak is 0.00002 and 0.0005 for each $50 \mathrm{mg} / \mathrm{dL}$ glucose concentration with FD measurements and direct TD measurements, respectively. The glucose concentration range of $25 \mathrm{mg} / \mathrm{dL}$ to $400 \mathrm{mg} / \mathrm{dL}$ is investigated, and the worst repeatability of this method is $3.65 \%$ for $300 \mathrm{mg} / \mathrm{dL}$.
\end{abstract}

Keywords: glucose concentration measurement; time-domain dielectric spectroscopy; microwave dielectric spectroscopy; ultra-wideband dielectric spectroscopy; dispersive medium analysis

\section{Introduction}

Diabetes Mellitus (DM) is a chronic, metabolic disease characterized by elevated levels of blood glucose, which leads over time to serious damage to the heart, blood vessels, eyes, kidneys, and nerves. About 422 million people worldwide have diabetes, and 1.6 million deaths are directly attributed to diabetes each year [1]. The normal glucose level in the blood ranges from $72 \mathrm{mg} / \mathrm{dL}$ to $108 \mathrm{mg} / \mathrm{dL}$, whereas patients with DM may experience levels as high as $400 \mathrm{mg} / \mathrm{dL}$ [2]. Having access to affordable treatment, including insulin, is critical for the survival of diabetics. Therefore, the exact knowledge of the blood glucose level is essential. Those who suffer from diabetes are supposed to measure their blood level several times a day with painful and invasive methods [3]. Currently, blood glucose levels are mostly monitored with ambulatory monitoring devices, where a drop of blood has to be drawn via a lancet and placed onto a chemically pre-treated strip inserted in a reading device [4]. Furthermore, the usual blood glucose monitoring for diabetics consists of three to six measurements per day with glucometers, which are invasive and need at least one drop of blood several times 
per day [5]. To overcome these problems, precise non-invasive glucose monitoring is in demand.

These days, electromagnetic waves have been used for various medical applications, including blood glucose measurement [2-12], skin cancer [13], and breast cancer [14]. Blood glucose measurement has been researched in different frequency ranges, from radiofrequency [11] to the infrared range [12]. Among methods that have been studied for non-invasive glucose monitoring, microwave spectroscopy (MS) is one of the most popular techniques. Because, as it was mentioned in [15], "in the microwave range, the electromagnetic waves can deeply penetrate the human tissue regions, where the main blood vessels are located". MS can be divided into two types based on their frequency range, wideband method, and resonant method. In wideband microwave spectroscopy (WBMS), material characteristics are determined by their complex dielectric permittivity in the microwave range. This approach has been proven to be highly valuable since the dielectric property of materials over a broad range of frequencies is unique for each material [16]. Therefore, a relatively straightforward material sensing and characterization can be performed with low to moderate cost integrated microwave radio hardware.

Most of the MS-based glucose-sensing research is based on the resonant method [2,8-10,15]. The whole concept of these references is measuring the resonant frequency shift by changing glucose concentration. Moreover, using the quality factor of the resonator instead of resonant frequency is another method for the characterization of the glucose and water solution. In $[8,10]$, detecting glucose levels up to about $270 \mathrm{mg} / \mathrm{dL}$ through fingerprints has been proposed using a microwave sensor and vector network analyzer (VNA). Researchers typically first establish sensing schemes for glucose in water and then advance further, studying glucose in the blood. The glucose contents of water were measured in the range of $0 \mathrm{mg} / \mathrm{dL}$ to $400 \mathrm{mg} / \mathrm{dL}$ in [9], with a sensitivity of $0.008 \mathrm{~dB}$ per $100 \mathrm{mg} / \mathrm{dL}$. Moreover, GlucoWise [17] follows an approach of a resonant sensor operating at $58 \mathrm{GHz}$ based on transmission techniques. For this aim, Gouzouasis et al. utilized two patch antennas and VNA for sensing the added glucose content of water [18]. In [18], it has been mentioned that "A change of $0.23 \mathrm{~dB}$ in transmission coefficient was achieved per $900 \mathrm{mg} / \mathrm{dL}$ glucose variation". Based on a monotonic relation, this is equivalent to a sensitivity of $0.005 \mathrm{~dB}$ for $18 \mathrm{mg} / \mathrm{dL}$ change. Since these results have been measured with VNA, a small VNA with a resolution of $0.005 \mathrm{~dB}$ will be required to make this sensor portable. In light of further measurement errors, this sensitivity is very weak. Furthermore, performing WBMS using co-planar and planar sensors has been introduced as a suitable approach for detecting glucose variation recently $[3,7,19]$. In all of these researches, the material under test (MUT) has to be in direct contact with the sensor. Consequently, the sensor's usage period will be short, which is not financially viable. Furthermore, contact-based measurement can be detrimental to MUT or the sensor in some situations. These issues can be solved by utilizing non-contact MS.

There are two methods for doing WBMS, the frequency domain (FD) and the time-domain (TD). The FD technique usually requires a high-cost and bulky VNA. In contrast with the conventional FD method, TD dielectric spectroscopy is cost, time, and space-efficient. However, some convenient setups were proposed recently in both FD and TD measurements [16,20-23]. A non-invasive time-domain WBMS system based on an artificial neural network for detecting glucose content of blood was proposed in [7]. A pair of custom-made ultra-wideband (UWB) rectangular patch bio-antennas were placed on a human earlobe, and TD transmission signals with a central frequency of $4.7 \mathrm{GHz}$ were measured using a transceiver [7]. Complex processing and a large duration of measurement are the weaknesses of this system. The proposed transmission setup of [24] is a kind of TD system in the 50 to $67 \mathrm{GHz}$ frequency range. It makes use of Discrete Fourier Transform (DFT) for processing TD transmitted data through the water with $50 \mathrm{mg} / \mathrm{dL}$ to $350 \mathrm{mg} / \mathrm{dL}$ glucose concentration. However, they just 
reported the difference between the DFT of different samples without any estimation for glucose concentration. Thus, a monotonic relation is not proven.

The main goal of this study is to compare the FD and TD methods for measuring the glucose concentration of aqueous solutions using WBMS. More precisely, TD transformed from an FD measurement versus a direct TD measurement has been studied. The proposed system is inspired by the previous research in which TDWBMS systems were presented for milk qualification [25,26]. In contrast to [25,26], in which permittivity of various milk was estimated using fast Fourier transform (FFT), a relationship between the measured reflected TD signal from MUT and its related glucose concentration is sought. First, the TD reflected signal from MUT has been calculated in MATLAB by applying electromagnetic theory equations. Then, the measurements have been conducted in a quasi-monostatic setup with direct FD and direct TD methods using VNA and Ilmsens radar [27], respectively. Water and glucose mixture has been used instead of the blood sample in all the measurements. It is worth mentioning that water has been used in various researches instead of the blood sample $[6,9,15,18,19,28,29]$. The change of blood plasma permittivity for various glucose concentrations is comparable with that of water for different glucose contents [30]. To the best of the author's knowledge, there is no TD-WBMS system for sensing the glucose content by processing data in TD. Therefore, a non-contact TD UWB setup for sensing the glucose concentration seems in demand.

The rest of this paper is organized as follows: data on the measured complex permittivity of water with various glucose concentration levels are reported in Section 2; in Section 3, TD reflection is calculated utilizing inverse Laplace Transform; Section 4 introduces the proposed setup for glucose concentration; comparison with previous studies and conclusions are presented in Sections 5 and 6, respectively.

\section{Electrical Properties of Aqueous Glucose Solution}

Since blood consists of a major amount of water, it can be assumed that its dielectric properties are related to those of aqueous glucose solutions [3]. The Cole-Cole model reported for blood plasma with different glucose concentrations clarifies that the changes in dielectric properties of blood and water are proportional to each other [30]. Therefore, measuring glucose concentration in water can lead to measuring blood glucose concentration in the early stages of research. Such an approach avoids asking for ethical approval from the ethics commission [31] at an early stage of research. The electromagnetic properties of water are required not only for calculating reflected signals from glucose solution but also for analyzing the results of the proposed setup.

The complex permittivity of glucose in water solution has been reported in the literature $[3,28]$. Although MUT temperature has a critical effect on permittivity, this effect was not considered in $[3,28]$. Therefore, the complex permittivity of glucose and $23{ }^{\circ} \mathrm{C}$ distilled water solution was measured with a commercial coaxial probe and VNA (Agilent N5244A PNA-X) from $10 \mathrm{MHz}$ to $20 \mathrm{GHz}$ frequency band. The measured real and imaginary parts of permittivity for water with different glucose content are plotted in Figure 1.

Water molecules are strongly polar in contrast with glucose with less polarity. Based on Figure 1, the real part of permittivity decreases, and the imaginary part of permittivity increases when we add sugar to water. This phenomenon is just for water. Both $\varepsilon^{\prime}$ and $\varepsilon^{\prime \prime}$ $\left(\varepsilon^{\prime}\right.$ and $\varepsilon^{\prime \prime}$ are real and imaginary parts of permittivity, respectively) increase with increasing the glucose concentration of water and $25 \mathrm{mg} / \mathrm{dL}$ glucose solution by the step of $50 \mathrm{mg} / \mathrm{dL}$. It is worth mentioning that this increment for $\varepsilon^{\prime \prime}$ continues till about $18 \mathrm{GHz}$ then $\varepsilon^{\prime \prime}$ follows the opposite trend. 


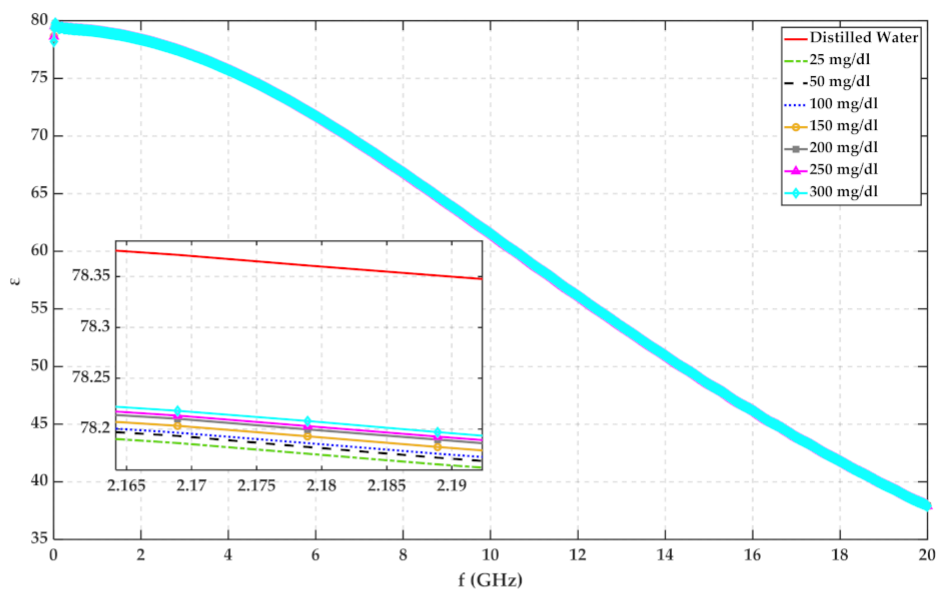

(a)

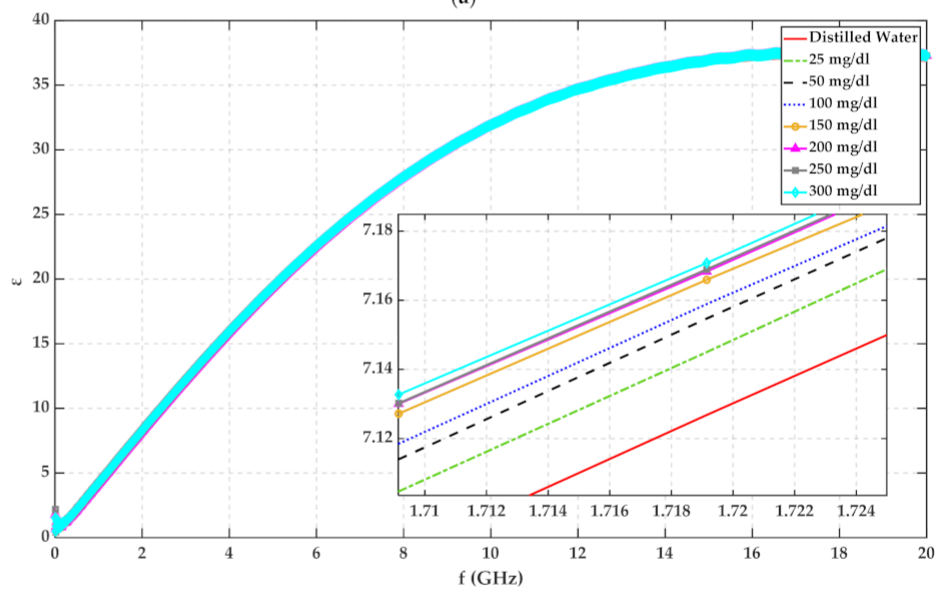

(b)

Figure 1. The measured permittivity of $23{ }^{\circ} \mathrm{C}$ distilled water with different glucose concentration (a) real part (b) imaginary part.

\section{Reflected Signal Calculation from Debye Medium}

The permittivity of water depends on frequency. In [32], it has been mentioned that "The Debye model commonly describes the electrical dispersion behavior of a homogeneous lossy media". This Model assumes the material dispersion as a linear effect. According to the first order Debye relaxation model, the complex permittivity $\varepsilon_{r}^{*}=\varepsilon^{\prime}-j \varepsilon^{\prime \prime}$ of dispersive materials can be presented with three parameters, as shown in (1). Where $\varepsilon_{s}$ and $\varepsilon_{\infty}$ are static and infinite permittivity, and $\tau$ is relaxation time [32].

$$
\varepsilon_{r}^{*}=\varepsilon_{\infty}+\frac{\varepsilon_{s}-\varepsilon_{\infty}}{1+j \omega \tau}
$$

For realizing how TD reflected signals change based on glucose concentration of aqueous solutions, the reflection coefficient $(\Gamma(t))$ of a Debye half-space in the time domain has been calculated using inverse Laplace transform. Reflection coefficient in Laplace domain can be written as (2) if a TE polarization plane wave $E^{i}(t)$ is incident on to a dispersive half-space from free space at an incidence angle $\theta$ relative to the normal to the interface [33]:

$$
\Gamma(s)=\frac{\cos \theta-\sqrt{\varepsilon_{r}(s)-\sin ^{2} \theta}}{\cos \theta+\sqrt{\varepsilon_{r}(s)-\sin ^{2} \theta}}
$$


For calculating inverse Laplace of a function, when $s \rightarrow \infty$ the function must approach zero. However, $\Gamma(s)$ approaches a non-zero value when $s \rightarrow \infty$. Therefore, $\bar{\Gamma}(s)=\Gamma(s)-\Gamma_{\infty}$ is defined where $\Gamma_{\infty}$ is [33]:

$$
\begin{gathered}
\Gamma_{\infty}=\lim _{s \rightarrow \infty} \Gamma(s)=\frac{1-K_{T E}}{1+K_{T E}} \\
K_{T E}=\frac{\sqrt{\varepsilon_{\infty}-\sin ^{2} \theta}}{\cos \theta}
\end{gathered}
$$

Rothwell calculated the $\bar{\Gamma}(t)$ based on (5) [33]:

$$
\frac{\bar{\Gamma}(t)}{F_{T E}}=s_{B} g_{1}(t)-2 s_{B}{ }^{2} \frac{K_{T E}}{1-K_{T E}{ }^{2}}\left[e^{-s_{A t}} u(t)\right] * g_{2}(t)
$$

where

$$
\begin{gathered}
g_{1}(t)=e^{-\frac{t}{\tau}}\left[\left(K_{T E}-1\right) \overline{I_{0}}\left(s_{B} \mathbf{t}\right)-\left(K_{T E}+1\right) \overline{I_{1}}\left(s_{B} \mathbf{t}\right)\right] \mathbf{u}(\mathbf{t}) \\
g_{2}(t)=e^{-\frac{t}{\tau}}\left[\left(K_{T E}-1\right) \overline{I_{0}}\left(s_{B} \mathbf{t}\right)+\left(K_{T E}+1\right) \overline{I_{1}}\left(s_{B} \mathbf{t}\right)\right] \mathbf{u}(\mathbf{t}) \\
F_{T E}=\frac{2 K_{T E}}{\left(1+K_{T E}\right)\left(1+K_{T E}{ }^{2}\right)} \\
s_{A}=\frac{1}{\tau} \frac{\varepsilon_{s}-1}{\varepsilon_{\infty}-1} \\
s_{B}=\frac{1}{2 \tau} \frac{\varepsilon_{s}-\varepsilon_{\infty}}{\varepsilon_{\infty}-\sin ^{2} \theta} \\
\overline{I_{n}}(x) \equiv e^{-\frac{t}{\tau}} I_{n}(x)
\end{gathered}
$$

where $I_{n}$ is modified Bessel Function of the first kind and order $\mathrm{n}$. The Debye parameters of glucose and $23{ }^{\circ} \mathrm{C}$ distilled water solution have been extracted in MATLAB using plots of Figure 1 and listed in Table 1 . Then, the reflected signal $E^{r}(t)$ according to $E^{r}(t)=E^{i}(t) * \Gamma(t)$ has been calculated in MATLAB for water with $50 \mathrm{mg} / \mathrm{dL}, 100 \mathrm{mg} / \mathrm{dL}$, and $150 \mathrm{mg} / \mathrm{dL}$ glucose when $E^{i}(t)$ is a Gaussian pulse as shown in Figure 2a. Reflected signals regarding different glucose concentrations have been plotted in Figure $2 b$.

Table 1. Extracted Debye parameter for $23^{\circ} \mathrm{C}$ water with the different glucose content.

\begin{tabular}{cccc}
\hline Glucose Concentration & $\boldsymbol{\varepsilon}_{\boldsymbol{s}}$ & $\boldsymbol{\varepsilon}_{\infty}$ & $\boldsymbol{\tau}($ in $\boldsymbol{p s})$ \\
\hline $50 \mathrm{mg} / \mathrm{dL}$ & 79.28 & 4.895 & 8.19 \\
$100 \mathrm{mg} / \mathrm{dL}$ & 79.29 & 4.897 & 8.20 \\
$150 \mathrm{mg} / \mathrm{dL}$ & 79.31 & 4.901 & 8.23 \\
\hline
\end{tabular}

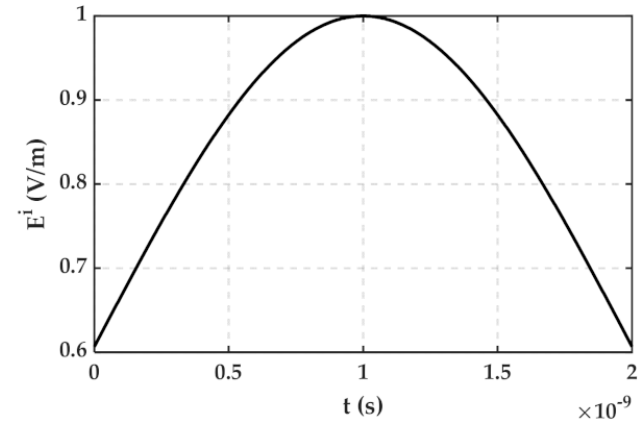

(a)

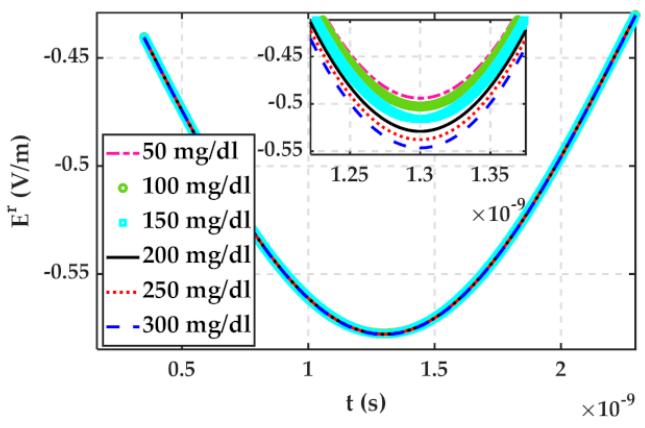

(b)

Figure 2. (a) Incident field to glucose concentration half-space medium (b) Reflected field from different glucose concentration medium. 
It can be seen that the depth of reflected signals in Figure $2 b$ decreases with increasing glucose concentration. Although an arbitrary shape dielectric object with a finite thickness plays the role of MUT in the proposed setup, modeling it as a dielectric half-space and ignoring higher-order reflections can be an acceptable assumption. Consider a dielectric object with thickness $l \geq 10 \mathrm{~mm}$, the time difference between first and second wall reflection is $\Delta t=\frac{2 \times l \times \sqrt{\left|\varepsilon_{r}^{*}\right|}}{c}$. In the microwave frequency band, $\sqrt{\left|\varepsilon_{r}^{*}\right|}$ for different glucose concentrations is more than 7 , then $\Delta t$ will be larger than 0.46 ns. In a UWB system with more than $2.5 \mathrm{GHz}$ bandwidth, this time difference is large enough for separating the first wall reflection. Water-based solutions are high loss dielectric, and their higher-order reflections can be neglected easily because of their small magnitude. Therefore, the same trend for TD reflected signals from glucose solution is expectable in simulations and measurements.

\section{Proposed Setup}

WBMS can be performed in either quasi-monostatic setup or bistatic setup. Usually, a quasi-monostatic setup requires two adjacent identical antennas for measuring reflected signals from MUT. Reversely, the MUT is placed between two antennas, and the transmission signal is recorded by the receiver in a bistatic method. In this section, a quasi-monostatic setup has been proposed for sensing the glucose concentration of aqueous solutions. The quasi-monostatic spectroscopy has the advantage of smaller required space for the whole system. Moreover, as water is a high loss material, the transmitted signal is weaker than the reflected signal. Therefore, an expensive analog to digital converter (ADC) with a higher resolution will be required for bistatic cases.

First, the setup has been simulated in CST studio suite full-wave software. Then, two different measurement methods have been utilized for proving the feasibility of TD signals for glucose sensing. In the former, FD measurement has been done with VNA and two Vivaldi antennas, and TD signals have been recorded by the transform option of VNA. The second one is a direct TD measurement in which a maximum length sequence (m-sequence) transceiver has been used for sensing MUT. These two setups have been operated in the frequency range of $0.1-6 \mathrm{GHz}$.

\subsection{Simulation Results}

Figure 3 shows the simulation setup in which transmitter and receiver antennas are two identical Vivaldi antennas. The distance between antennas is $80 \mathrm{~mm}$. To have a compact setup, the required space has been chosen to be as small as possible. The radiative near-field region for an antenna with maximum dimension $\mathrm{D}$ at the wavelength of $\lambda$ is defined based on (12):

$$
0.62 \times \sqrt{\frac{D^{3}}{\lambda}} \leq \text { radiative near }- \text { field region } \leq \frac{2 \times D^{2}}{\lambda}
$$

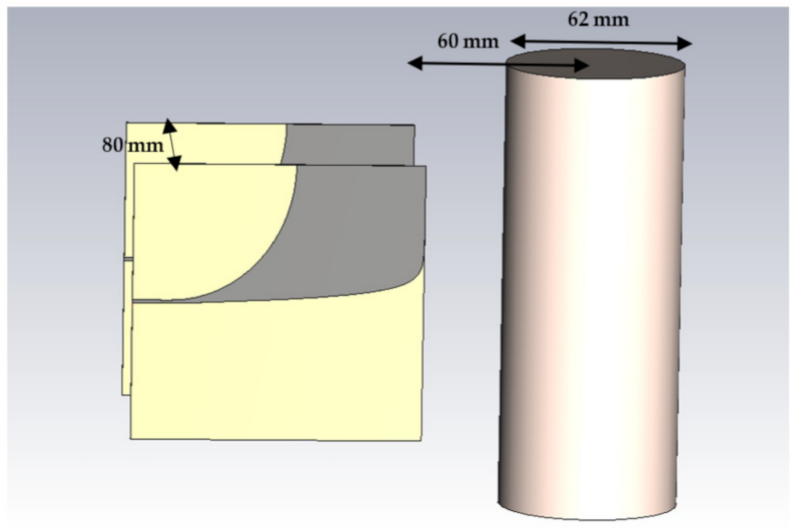

Figure 3. Simulated quasi-monostatic setup. 
For an antenna with the maximum dimension of $100 \mathrm{~mm}$ at $2.9 \mathrm{GHz}$ central frequency, this region will be from $60 \mathrm{~mm}$. Hence, the center of $62 \mathrm{~mm}$ diameter MUT is located $60 \mathrm{~mm}$ from the antennas. On the other hand, the effect of the surrounding medium will rise with increasing the distance between MUT and antennas.

The container thickness is $2 \mathrm{~mm}$, and its material has been chosen as lossy quartz with a dielectric constant and loss tangent of 3.75 and 0.0004 , respectively. Measured complex permittivity of Section 2 has been imported to CST for defining dispersion characteristics of MUTs. The simulation has been operated in the frequency range of $0.1 \mathrm{GHz}$ to $6 \mathrm{GHz}$. The simulation results for water with different glucose concentrations are plotted in Figure 4.

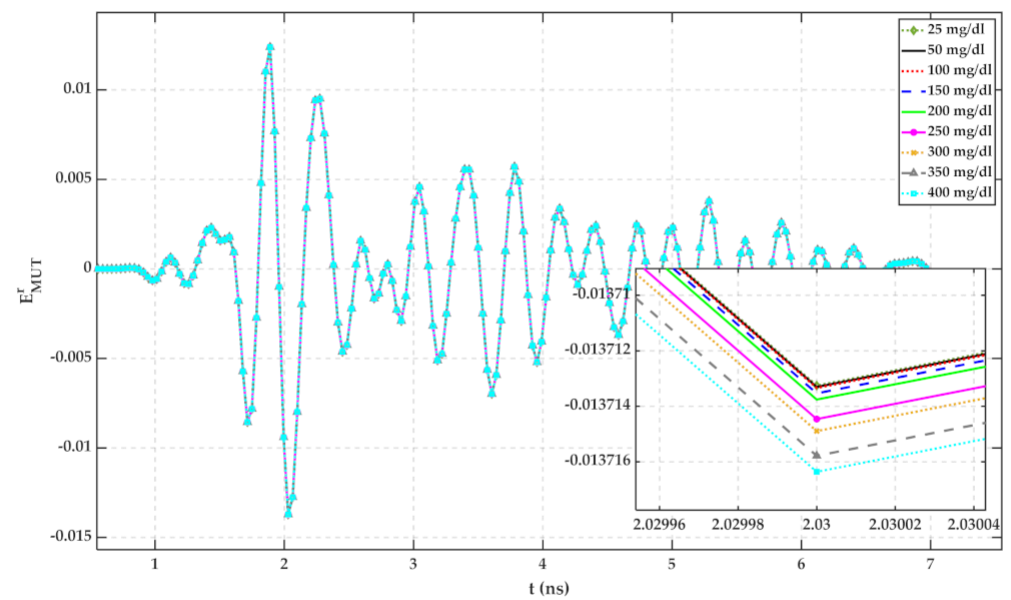

Figure 4. Simulation result for reflected signals from water with the different glucose content.

Based on Figure 4, the reflected signal increases with increasing glucose content. Hence, it can be seen that the glucose content of water is distinguishable with measuring TD reflected signals. For ignoring the mutual coupling between the antennas and the effect of the container, signals regarding different MUTs are subtracted from signals related to $25 \mathrm{mg} / \mathrm{dL}$ and shown in Figure 5. In this paper, water with $25 \mathrm{mg} / \mathrm{dL}$ glucose has been utilized as a reference signal because, based on Figure 1, the permittivity of water does not follow the same trend as other samples. In Figure $1 \mathrm{~b}$, the imaginary part of the water is close to that of $175 \mathrm{mg} / \mathrm{dL}$. It seems, by adding a small amount of glucose to water, its electrical properties change.

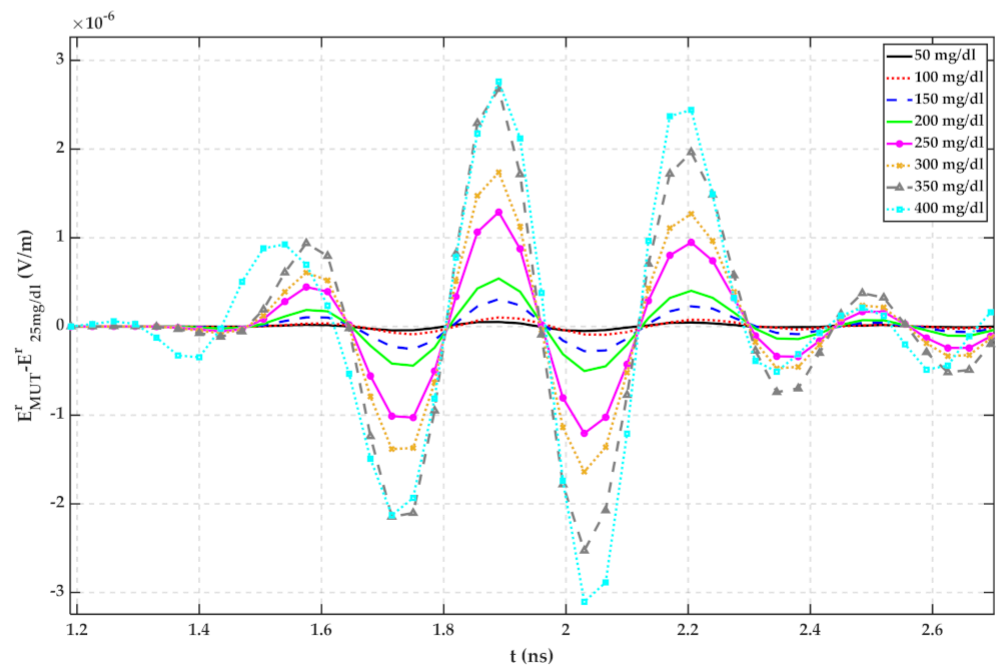

Figure 5. Simulation result for reflected signal differences of various MUTs with respect to water with $25 \mathrm{mg} / \mathrm{dL}$ glucose. 


\subsection{Frequency Domain Measurement Results}

The measurement configuration is shown in Figure 6. All the details are the same as with the simulation setup. The measurements of this section have been conducted along with measurements of Section 2 to examine the effect of the electrical properties of the liquid on its reflected signal. The Agilent N5244A PNA-X has been utilized for measuring the S-parameter and transforming them to TD data. First, the measurement has been taken on the empty setup when the container is not in place. The measured scattering parameters of the empty setup for 201 frequency samples have been illustrated in Figure 7. It can be seen that the antenna has an acceptable matching over the desired frequency band of 0.1-6 GHz.

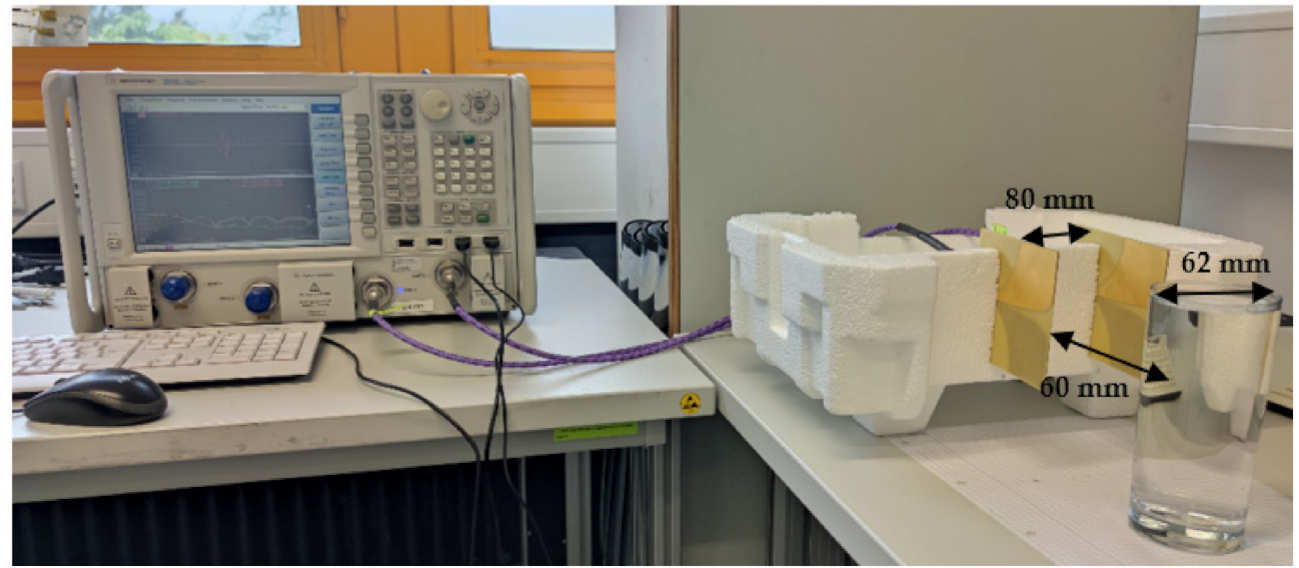

Figure 6. Proposed FD measurement setup.

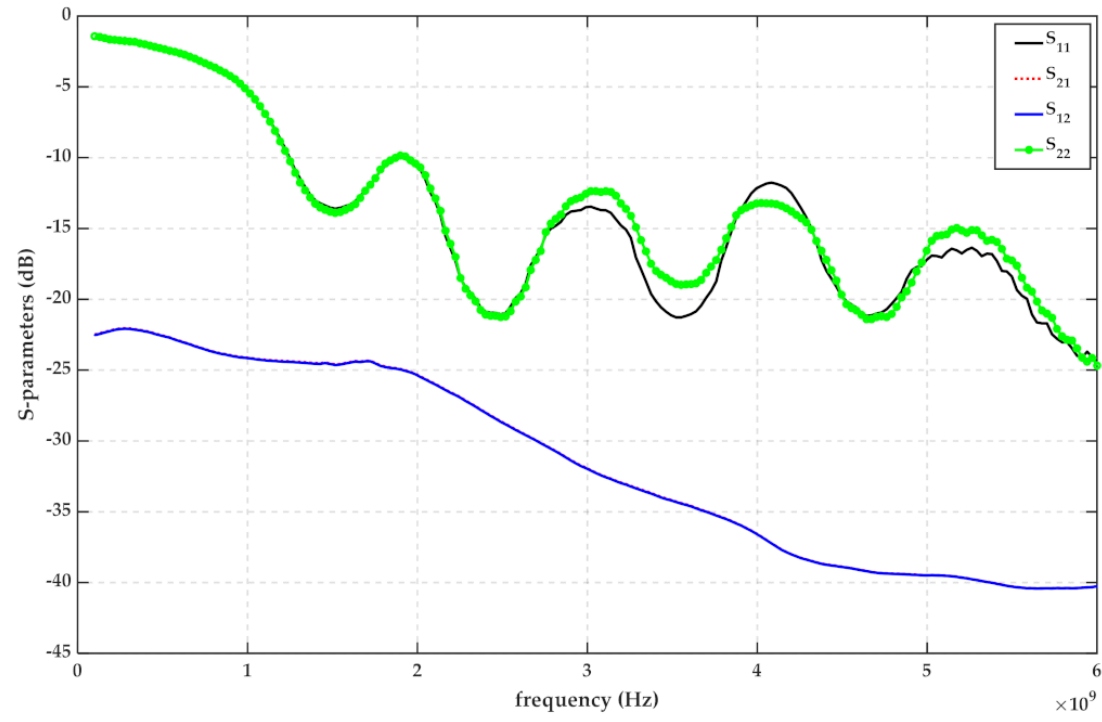

Figure 7. Measured scattering parameters of the empty setup.

In each step, $200 \mathrm{mg}$ glucose has been weighed with a $1 \mathrm{mg}$ accuracy scale and added to 0.4 -L $23{ }^{\circ} \mathrm{C}$ distilled water. $S_{21}$ of the bottle full of water and glucose solution has been measured with VNA. Figure 8 shows $S_{21}$ for water with various glucose contents. Based on Figure 8, no feasible relationship has been found between the $S_{21}$ and glucose concentrations on the whole frequency band. The discrete inverse z-transform of $S_{21}$ performed by VNA for various MUTs has been plotted in Figure 9. 


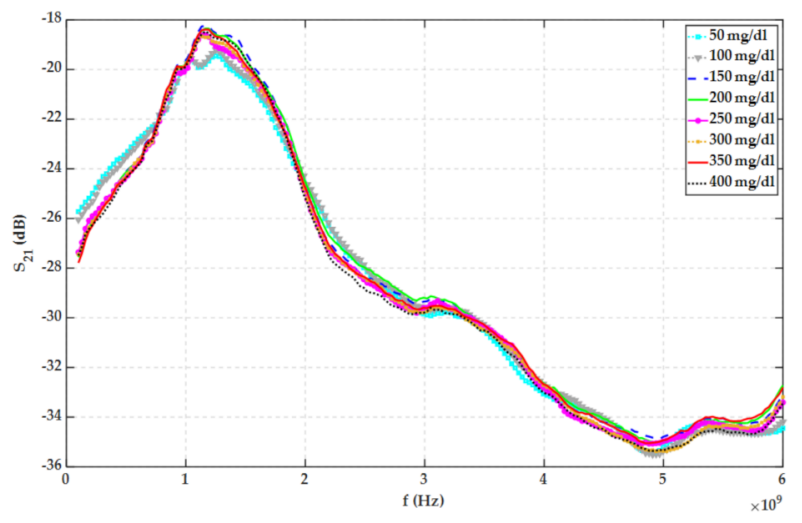

Figure 8. Measured scattering parameters regarding water with the different glucose content.

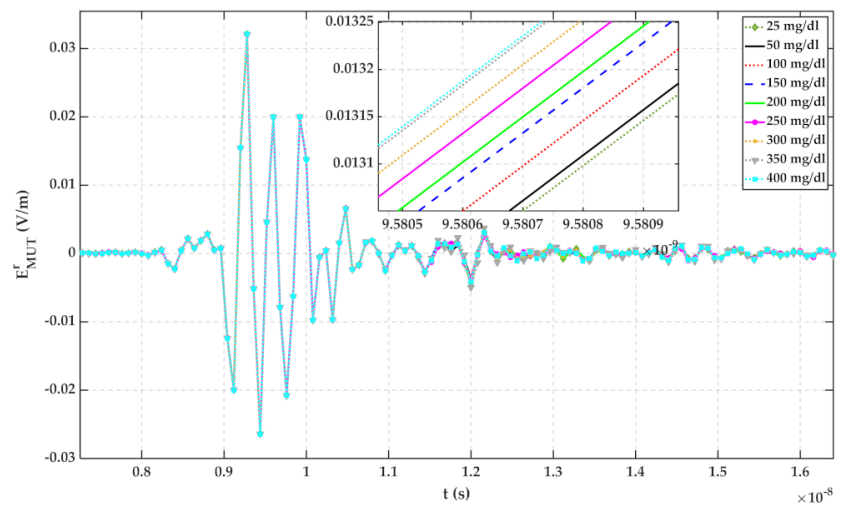

Figure 9. Transformed TD reflected signals regarding different MUTs (measured with PNA-X).

According to Figure 9, the more the glucose content of water, the larger the height of the peak of the reflected signal. This can be justified based on the results of Figures 2 and 4 . To estimate the glucose concentration of water according to reflected TD signals, the signals regarding water and glucose solutions have been subtracted from the signal obtained from water with $25 \mathrm{mg} / \mathrm{dL}$ glucose content and plotted in Figure 10. As can be seen by comparing signals of Figures 9 and 10, the signals up to 9.2 ns are mutual coupling between antennas, and the signals after $11.5 \mathrm{~ns}$ are the signals reflected from the wall and other existing things in the laboratory. Therefore, the signals from about $9.8 \mathrm{~ns}$ to $11.5 \mathrm{~ns}$ can be considered as the response of MUTs to the incident wave.

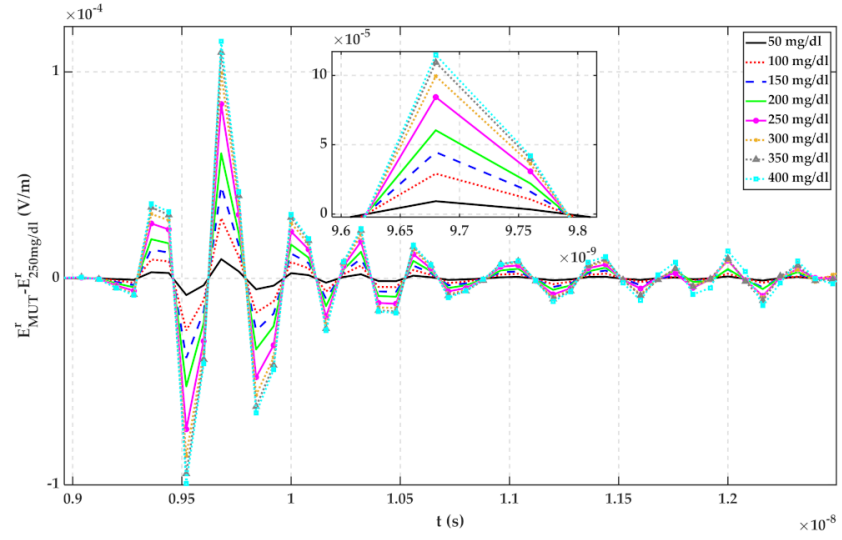

Figure 10. Reflected signal differences of various MUTs concerning water with $25 \mathrm{mg} / \mathrm{dL}$ glucose (measured with PNA-X). 


\subsection{Time Domain Measurement Results}

In this section, the measurements have been done using Ilmsens radar, as shown in Figure 11, in the frequency range of $0.1-6 \mathrm{GHz}$. The transmitted power and time resolution of this sensor are about $-7 \mathrm{dBm}$ and $78 \mathrm{ps}$, respectively. All dimensions are the same as in Section 4.2. This radar is a UWB m-sequence transceiver.
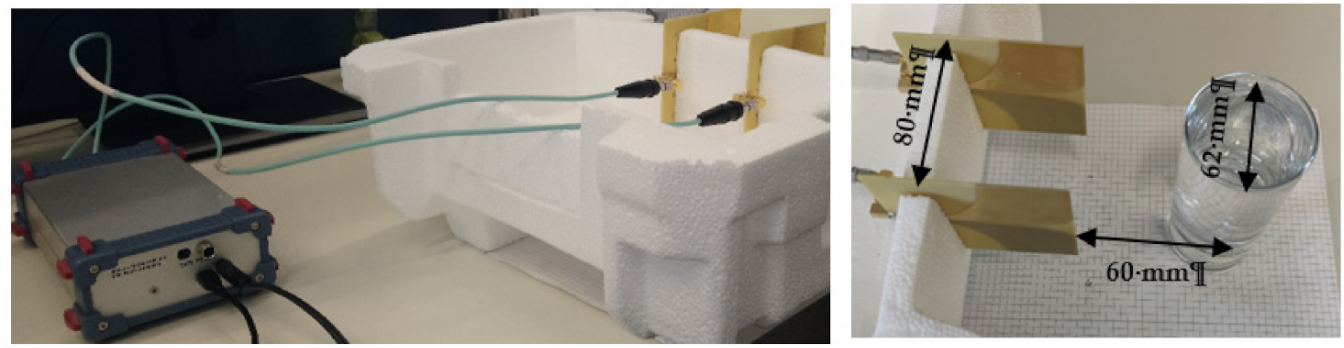

Figure 11. Measurement setup with Ilmsens radar.

The block diagram of the m-sequence radar is shown in Figure 12. Typical UWB radars require sub-nanosecond pulse or frequency modulated continuous wave (FMCW) chirps for their operation. Sub-nanosecond pulse can be generated by step recovery diode (SRD). This method suffers from a high peak-to-average power ratio, and the FMCW method has high power consumption and a limited frequency band [34]. To address these problems, an $\mathrm{m}$-sequence transceiver is used. The $\mathrm{m}$-sequence is generated by a digital shift register, which is pushed by a stable RF-clock with frequency $f_{c}$ [34]. The spectral band of $\left[0 f_{c}\right]$ contains 80 percent of the total signal energy of the m-sequence. This drastically reduces the stress caused by strong electric fields, which the MUT would be exposed to due to the small volume of interaction [34].

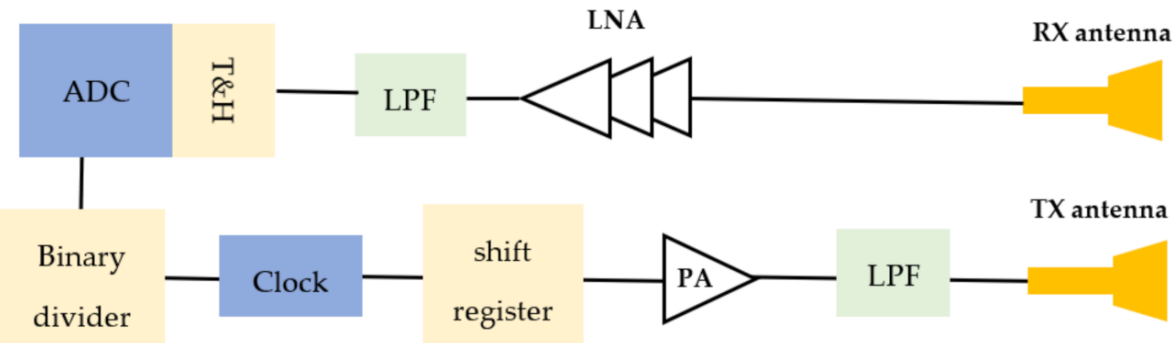

Figure 12. Block diagram of the m-sequence radar.

Same as Section 4.2, the MUT is a mixture of $23{ }^{\circ} \mathrm{C}$ distilled water with different amounts of glucose. Reflected signals in the time domain have been recorded by a computer. Each MUT measurement has been repeated 10 times to verify the repeatability of the measurement. The average of reflected signals from each MUT has been calculated and plotted in Figure 14. The signal from up to $10 \mathrm{~ns}$ is the internal leakage of the radar.

Plots of Figure 14 have been calculated by subtracting reflected signals of MUTs from water with $25 \mathrm{mg} / \mathrm{dL}$ glucose content. The standard deviation (std) of 10 measurements for each MUT has been calculated using (13) and is shown in Figure 13. For a random variable vector A made up of $\mathrm{k}$ scalar observations with the mean of $\mu$, the standard deviation is defined as:

$$
\operatorname{std}=\sqrt{\frac{1}{k} \sum_{i=1}^{k}\left|A_{i}-\mu\right|^{2}}
$$

It can be seen from both figures that the reflected signal regarding more glucose content has a larger peak height. 


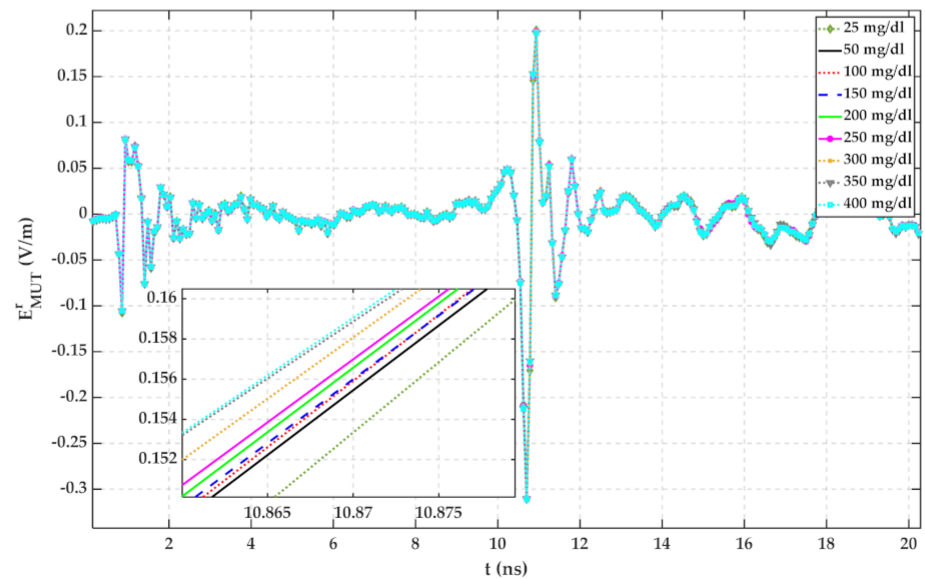

Figure 13. Reflected signal differences of various MUTs concerning water with $25 \mathrm{mg} / \mathrm{dL}$ glucose (measured with m-sequence radar).

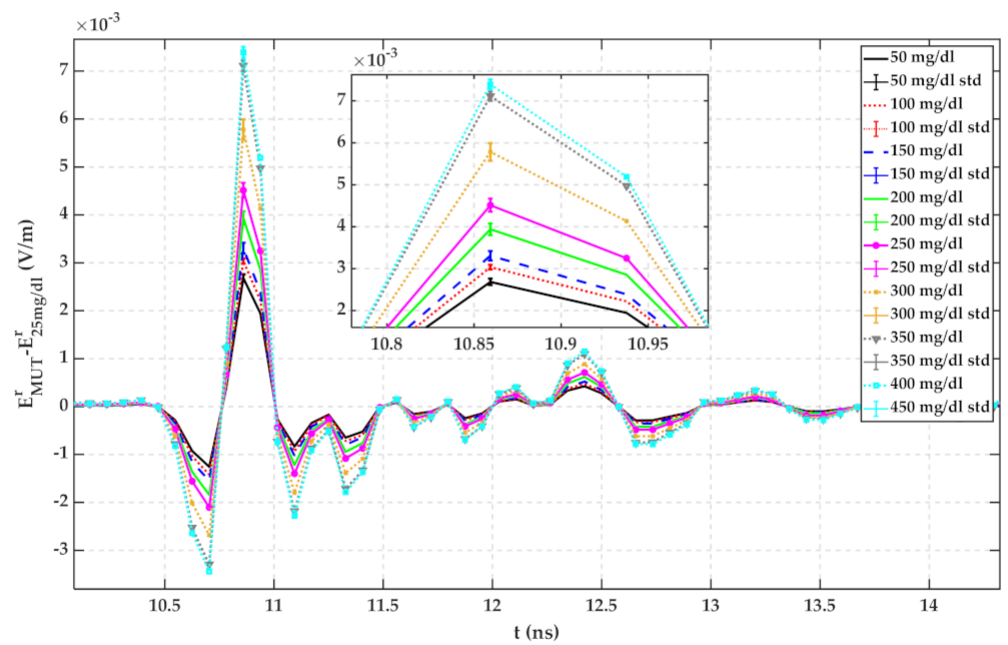

Figure 14. TD reflected signals regarding different MUTs (measured with m-sequence radar).

\subsection{Mapping the Glucose Concentration of Water to the Maximum of Reflected Signals}

For estimating the glucose content of water, a monotonic relation between the height of the peaks of signals in Figures 10 and 13 and the corresponding glucose concentration has been performed and illustrated in Figure 15. The root means square error (RMSE) of both fits is below 0.0017 . For example, it can be extracted from Figure $15 \mathrm{~b}$ if the maximum of the reflected signal was 0.003 , the glucose content of water would be $100 \mathrm{mg} / \mathrm{dL}$.

Although this paper is an early step study of this method, the minimum amount of glucose that is detectable using this method is $50 \mathrm{mg} / \mathrm{dL}$. Although $60 \mathrm{mg} / \mathrm{dL}$ is commonly cited as the lower limit of normal glucose, symptoms of hypoglycemia usually do not occur until $50 \mathrm{mg} / \mathrm{dL}$ [35]. Hence, this limit of detection is acceptable. The operating range for this method is $25 \mathrm{mg} / \mathrm{dL}$ to $400 \mathrm{mg} / \mathrm{dL}$. Indeed, based on the measurements, this method can work up to $1000 \mathrm{mg} / \mathrm{dL}$. However, more measurements and statistical data will be needed; that is the purpose of future study. The sensitivity of FD and TD methods are 0.00002 and 0.0005 per $50 \mathrm{mg} / \mathrm{dL}$, respectively. The repeatability of the measurements has been calculated based on (14). The minimum of repeatability is $1.6 \%$ for $400 \mathrm{mg} / \mathrm{dL}$, and the maximum is $3.6 \%$ for $300 \mathrm{mg} / \mathrm{dL}$.

$$
\% \text { Repeatability }=\frac{\text { std }}{\mu} \times 100
$$




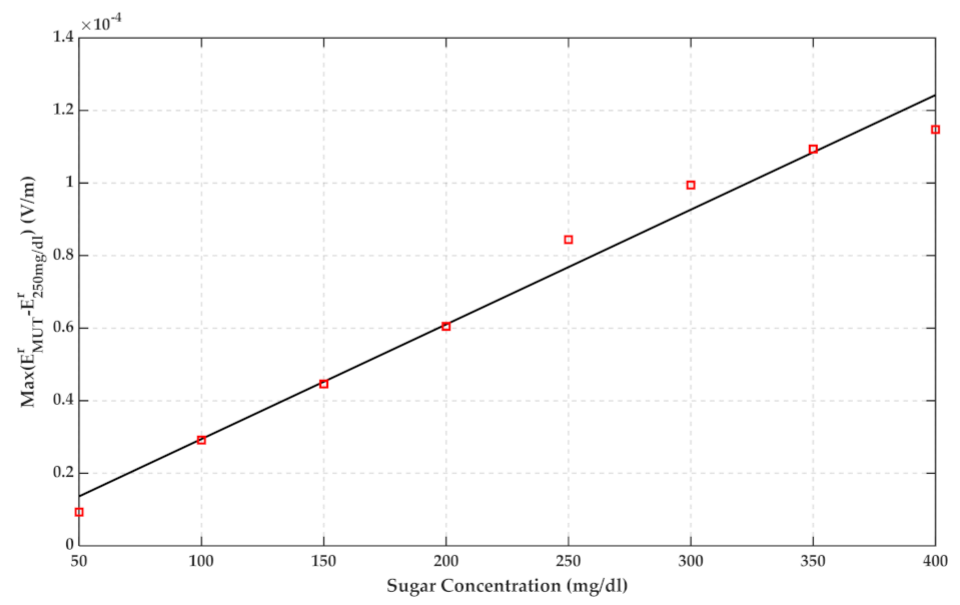

(a)

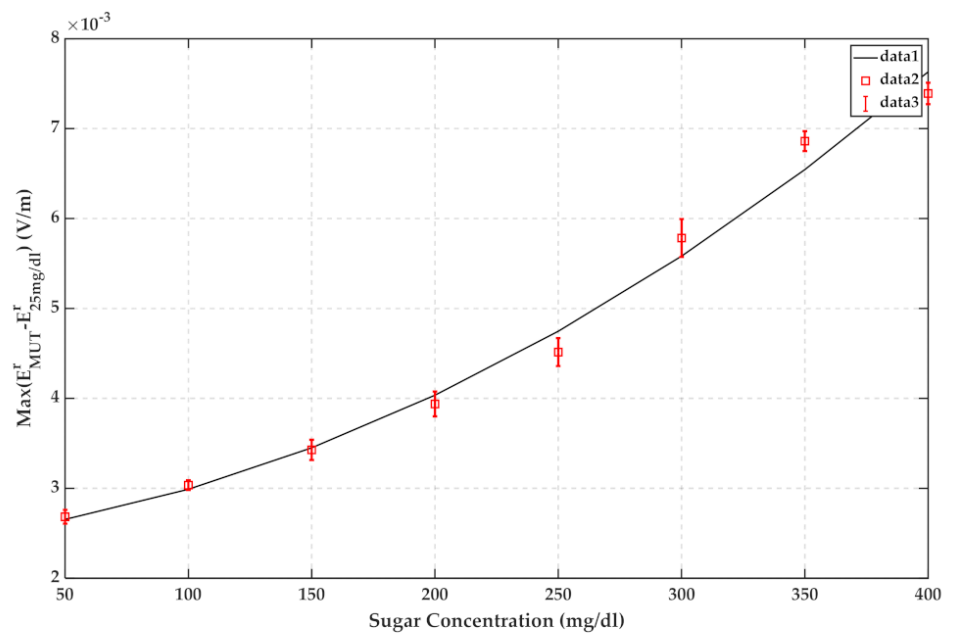

(b)

Figure 15. Maximum of reflected signals and corresponding linear fits as a function of glucose concentration (a) VNA measurement result (b) m-sequence radar measurement result.

\section{Discussion}

TD microwave dielectric spectroscopy was shown to be promising for measuring the glucose concentration of the aqueous solution. As mentioned in this paper, although there was no relationship between $S_{21}$ of the various MUTs, the peak heights of the TD signals were mapped to their related glucose content. However, some research reports the change of the S-parameter with glucose concentration using the resonance method, but the sensitivity is weak and needs an accurate bulky VNA. Using TD signals, coupling, multiple reflections, and multipath effects were observed separately from the MUT. Based on this paper, TD dielectric spectroscopy measurement can be done in either the FD or TD method. However, interpreting data regarding the glucose content in TD is much easier. Therefore, if one does the measurement in FD, it is advisable to transform the data to TD for better interpretation. Inverse z-transform is required for calculating TD signals from the measured S-parameter. Nevertheless, TD measurement usually is convenient and has the potential of being portable. An acceptable sensitivity for measuring the glucose content of water is reachable with direct TD measurement, as shown in Section 4.3. Using a water and glucose mixture instead of the blood sample can be a reliable approximation for the early step of research since, according to reflection theory, the magnitude of permittivity of MUT is the only parameter that affects the reflected signal from the medium. The change of blood plasma permittivity for various glucose concentrations is comparable with that of water for different glucose contents. 
According to Figure 5, Figure 6, Figure 7, Figure 8, Figure 9, Figure 10, the reflected signal differences are more obvious in the measurement than in the simulation. CST fitted the imported complex permittivity of dispersive materials with the Debye model. However, the Debye model is the approximation of linear dielectric properties of dispersive materials, and in the real world, we may face different challenges for testing aqueous solutions [29]. According to Figure 10, Figure 11, Figure 12, Figure 14, reflected signal differences for m-sequence radar measurement results are much larger than that of VNA measurement. This happened because the m-sequence receiver amplifies the captured signal with a gain of about $15 \mathrm{~dB}$. Furthermore, it is proof of the claim that glucose sensing can be done with a small transceiver with acceptable accuracy.

One may argue that higher-order reflection can affect reflected signals if a small container is used. However, it was mentioned in the previous paper that the second wall reflection does not have any effect on the measurement data for a 6 -cm diameter container [26]. Based on Section 3, with a bandwidth of more than $2.5 \mathrm{GHz}$, the second wall reflection is separable from TD signals if the water is placed in a bottle with more than $1 \mathrm{~cm}$ diameter.

Table 2 compares the proposed non-contact wideband TD setup with the previously reported works in the area of glucose concentration measurement. In [7] is a low-cost TD setup in which glucose concentration was measured through the earlobe with a max error of $16 \mathrm{mg} / \mathrm{dL}$. The weaknesses of this setup are complex processing and being timeconsuming. Sensing glucose level with an FD resonant method utilizing VNA and coplanar waveguide was presented in $[8,10]$. These two setups required an accurate and expensive VNA that could detect about $1.34 \mathrm{MHz}$ resonant shift per mg/dL. Moreover, the early research on the Glucowise sensor was presented in [18]. This sensor needs a VNA which can distinguish $0.005 \mathrm{~dB}$ change for the magnitude of transmission coefficient per $18 \mathrm{mg} / \mathrm{dL}$ glucose variation. In [24], TD measurement was conducted on the water glucose solution. The changes in DFT of TD data were reported for various glucose concentrations. However, there was not a clear estimation for detecting glucose.

Table 2. Summary and comparison with related work.

\begin{tabular}{|c|c|c|c|c|c|}
\hline Ref. & Setup Properties & Frequency Range & MUT Type & Method & Price \\
\hline [7] & UWB planar antenna, transceiver & $3.23-12 \mathrm{GHz}$ & Human Earlobe & Direct TD & Low \\
\hline [8] & coplanar waveguide and VNA & $4.5 \mathrm{GHz}$ and $7.5 \mathrm{GHz}$ & $\begin{array}{c}\text { Finger for } 89-262 \mathrm{mg} / \mathrm{dL} \\
\text { glucose level }\end{array}$ & Direct FD & High \\
\hline [10] & coplanar waveguide and VNA & $1.95-2.2 \mathrm{GHz}$ & $\begin{array}{c}\text { Finger for } 90-190 \mathrm{mg} / \mathrm{dL} \\
\text { glucose level }\end{array}$ & Direct FD & High \\
\hline [18] & VNA and patch Antenna & $58 \mathrm{GHz}$ & $\begin{array}{l}\text { Water with } 0-278 \mathrm{mg} / \mathrm{dL} \\
\text { glucose content }\end{array}$ & Direct FD & High \\
\hline [24] & $\mathrm{mm}$-wave radar and $\mathrm{PC}$ & $57-64 \mathrm{GHz}$ & $\begin{array}{l}\text { Water with } 0-350 \mathrm{mg} / \mathrm{dL} \\
\text { glucose content }\end{array}$ & FD transformed from TD & Medium \\
\hline This work & Transceiver and UWB antenna & $1-10 \mathrm{GHz}$ & $\begin{array}{l}\text { Water with } 25-225 \\
\mathrm{mg} / \mathrm{dL} \text { glucose content }\end{array}$ & $\begin{array}{l}\text { Direct TD and TD } \\
\text { transformed from FD }\end{array}$ & Low \\
\hline
\end{tabular}

\section{Conclusions}

In this paper, early research on TD analysis for detecting glucose concentration of the aqueous solution was presented. A comparison between FD and TD measurement results and their data analysis was presented. Inverse Laplace transform was served for calculating reflected signals from a Debye medium. No matter whether the measurements were done using the FD or TD method, there was a monotonic relationship between the maximum of TD data and their associated glucose concentrations. The change of peak height of the TD reflected signal is about 0.00002 for $50 \mathrm{mg} / \mathrm{dL}$ glucose concentration with FD measurements and 0.0005 with direct TD measurements. This magnitude resolution is reachable with a cheap 12 bit analog to digital converter in the receiver part. Measurement results showed good agreement with calculated numerical data in MATLAB. In comparison with the previous studies, this method has the potential of being portable, non-contact, and convenient. 
One of the critical factors for characterizing liquids is temperature. Since the permittivity of liquids is sensitive to temperature, it is important to keep the temperature constant. To get more accurate results, doing measurements at a controllable temperature is one of our future study aims. Since this method is an early research study for sensing the glucose content of the blood, the proposed method must be modified in the future for in-vivo cases. Moreover, fabricating a low-cost and portable transceiver is another direction that authors would intend to follow.

Author Contributions: Conceptualization: S.S. and G.F.; Data curation: S.S., S.C. and G.F.; Methodology: S.S. and S.C.; Software: S.S.; Validation: S.S., G.F.; Formal analysis: S.S.; Investigation: S.S.; Writing—original draft preparation: S.S.; Writing—review and editing: S.C. and G.F.; Visualization: S.S.; Supervision: G.F.; Project administration: G.F.; All authors have read and agreed to the published version of the manuscript.

Funding: This research received no external funding.

Institutional Review Board Statement: Not applicable.

Informed Consent Statement: Not applicable.

Data Availability Statement: The data that support the findings of this study are available on request from authors.

Acknowledgments: The authors would like to acknowledge their special thanks to Jürgen Sachs and Ilmsens company for providing m-sequence radar for their measurement. The authors would like to express their special thanks to Jens Kirchner, Medical Electronics \& Multiphysics Systems (MEMPS) group, and Max Bartunik, a member of MEMPS group, for supporting them during their research. The authors also would like to thank the German Academic Exchange Service (DAAD) for supporting the collaboration of authors at the University of Erlangen-Nuremberg.

Conflicts of Interest: The authors declare no conflict of interest.

\section{Abbreviations}

$\begin{array}{ll}\text { WBMS } & \text { Wideband microwave spectroscopy } \\ \text { TD } & \text { Time-domain } \\ \text { FD } & \text { Frequency domain } \\ \text { m-sequence } & \text { Maximum length sequence } \\ \text { DM } & \text { Diabetes Mellitus } \\ \text { MS } & \text { Microwave spectroscopy } \\ \text { VNA } & \text { Vector network analyzer } \\ \text { MUT } & \text { Material under test } \\ \text { UWB } & \text { Ultra-wideband } \\ \text { DFT } & \text { Discrete Fourier Transform } \\ \text { FFT } & \text { Fast Fourier transform } \\ \text { ADC } & \text { Analog to digital converter } \\ \text { FMCW } & \text { Frequency modulated continuous wave } \\ \text { SRD } & \text { Step recovery diode } \\ \text { std } & \text { Standard deviation } \\ \text { RMSE } & \text { The root means square error }\end{array}$

\section{References}

1. Roglic, G. Available online: https:/www.who.int/health-topic/diabetes (accessed on 20 January 2022).

2. Turgul, V.; Kale, I. Permittivity extraction of glucose solutions through artificial neural networks and non-invasive microwave glucose sensing. Sens. Actuators A Phys. 2018, 277, 65-72. [CrossRef]

3. Hofmann, M.; Fischer, G.; Weigel, R.; Kissinger, D. Microwave-based noninvasive concentration measurements for biomedical applications. IEEE Trans. Microw. Theory Tech. 2013, 61, 2195-2204. [CrossRef]

4. Bruen, D.; Delaney, C.; Florea, L.; Diamond, D. Glucose sensing for diabetes monitoring: Recent developments. Sensors 2017, 17, 1866. [CrossRef] [PubMed] 
5. Hofmann, M.; Fersch, T.; Weigel, R.; Fischer, G.; Kissinger, D. A novel approach to non-invasive blood glucose measurement based on RF transmission. In Proceedings of the 2011 IEEE International Symposium on Medical Measurements and Applications, Bari, Italy, 30-31 May 2011; pp. 39-42. [CrossRef]

6. Omer, A.E.; Gigoyan, S.; Shaker, G.; Safavi-Naeini, S. WGM-Based Sensing of Characterized Glucose- Aqueous Solutions at mm-Waves. IEEE Access 2020, 8, 38809-38825. [CrossRef]

7. Islam, M.; Ali, M.S.; Shoumy, N.J.; Khatun, S.; Karim, M.S.A.; Bari, B.S. Non-invasive blood glucose concentration level estimation accuracy using ultra-wide band and artificial intelligence. SN Appl. Sci. 2020, 2, 278. [CrossRef]

8. Kiani, S.; Rezaei, P.; Fakhr, M. Dual-Frequency Microwave Resonant Sensor to Detect Noninvasive Glucose-Level Changes Through the Fingertip. IEEE Trans. Instrum. Meas. 2021, 70, 6004608. [CrossRef]

9. Jang, C.; Park, J.-K.; Lee, H.-J.; Yun, G.-H.; Yook, J.-G. Non-Invasive Fluidic Glucose Detection Based on Dual Microwave Complementary Split Ring Resonators with a Switching Circuit for Environmental Effect Elimination. IEEE Sens. J. 2020, 20, 8520-8527. [CrossRef]

10. Cebedio, M.C.; Rabioglio, L.A.; Gelosi, I.E.; Ribas, R.A.; Uriz, A.J.; Moreira, J.C. Analysis and Design of a Microwave Coplanar Sensor for Non-Invasive Blood Glucose Measurements. IEEE Sens. J. 2020, 20, 10572-10581. [CrossRef]

11. Caduff, A.; Hirt, E.; Feldman, Y.; Ali, Z.; Heinemann, L. First human experiments with a novel non-invasive, non-optical continuous glucose monitoring system. Biosens. Bioelectron. 2003, 19, 209-217. [CrossRef]

12. Tarr, R.V.; Steffes, P.G. Non-Invasive Blood Glucose Measurement System and Method Using Stimulated Raman Spectroscopy U.S. Patent 5243983, 14 September 1993.

13. Mehta, P.; Chand, K.; Narayanswamy, D.; Beetner, D.G.; Zoughi, R.; Stoecker, W.V. Microwave reflectometry as a novel diagnostic tool for detection of skin cancers. IEEE Trans. Instrum. Meas. 2006, 55, 1309-1316. [CrossRef]

14. Porter, E.; Bahrami, H.; Santorelli, A.; Gosselin, B.; Rusch, L.A.; Popovic, M. A Wearable Microwave Antenna Array for Time-Domain Breast Tumor Screening. IEEE Trans. Med. Imaging 2016, 35, 1501-1509. [CrossRef] [PubMed]

15. Zeising, S.; Kirchner, J.; Khalili, H.F.; Ahmed, D.; Lubke, M.; Thalmayer, A.; Fischer, G. Towards Realisation of a Non-Invasive Blood Glucose Sensor Using Microstripline. In Proceedings of the 2020 IEEE International Instrumentation and Measurement Technology Conference (I2MTC), Dubrovnik, Croatia, 25-28 May 2020; pp. 1-6. [CrossRef]

16. Ghiri, R.E.; Saghati, A.P.; Kaya, E.; Entesari, K. A Miniaturized Contactless UWB Microwave System for Time-Domain Dielectric Spectroscopy. IEEE Trans. Microw. Theory Tech. 2017, 65, 5334-5344. [CrossRef]

17. Glucowise. Available online: http://www.gluco-wise.com/ (accessed on 20 January 2022).

18. Gouzouasis, I.; Cano-Garcia, H.; Sotiriou, I.; Saha, S.; Palikaras, G.; Kosmas, P.; Kallos, E. Detection of varying glucose concentrations in water solutions using a prototype biomedical device for millimeter-wave non-invasive glucose sensing. In Proceedings of the 2016 10th European Conference on Antennas and Propagation (EuCAP), Davos, Switzerland, 10-15 April 2016; pp. 1-4 [CrossRef]

19. Xiao, X.; Li, Q. A Noninvasive Measurement of Blood Glucose Concentration by UWB Microwave Spectrum. IEEE Antennas Wirel. Propag. Lett. 2017, 16, 1040-1043. [CrossRef]

20. Nasr, I.; Nehring, J.; Aufinger, K.; Fischer, G.; Weigel, R.; Kissinger, D. Single-and dual-port 50-100-GHz integrated vector network analyzers with on-chip dielectric sensors. IEEE Trans. Microw. Theory Tech. 2014, 62, 2168-2179. [CrossRef]

21. Helmy, A.A.; Entesari, K. A 1-8-GHz miniaturized spectroscopy system for permittivity detection and mixture characterization of organic chemicals. IEEE Trans. Microw. Theory Tech. 2012, 60, 4157-4170. [CrossRef]

22. Zeng, X.; Fhager, A.; He, Z.; Persson, M.; Linner, P.; Zirath, H. Development of a time domain microwave system for medical diagnostics. IEEE Trans. Instrum. Meas. 2014, 63, 2931-2939. [CrossRef]

23. Ghiri, R.E.; Entesari, K. A Miniaturized 3-10GHz Dual-Comb Spectroscopy System for Chemical Detection. In Proceedings of the 2019 IEEE MTT-S International Microwave Symposium (IMS), Boston, MA, USA, 2-7 June 2019; pp. 1492-1494. [CrossRef]

24. Omer, A.E.; Safavi-Naeini, S.; Hughson, R.; Shaker, G. Blood glucose level monitoring using an FMCW millimeter-wave radar sensor. Remote Sens. 2020, 12, 385. [CrossRef]

25. Saeedi, S.; Chammani, S. Milk Characterization Using Time Domain Wide Band Dielectric Spectroscopy. In Proceedings of the 2020 IEEE International Symposium on Antennas and Propagation and North American Radio Science Meeting, Montreal, QC, Canada, 5-10 July 2020; pp. 833-834. [CrossRef]

26. Saeedi, S.; Chammani, S. Non-contact Time Domain Ultra Wide Band Milk Spectroscopy. IEEE Sens. J. 2021, 21, 13849-13857. [CrossRef]

27. Ilmsens GmbH. Available online: https:/ / www.ilmsens.com/products/m-explore/ (accessed on 20 January 2022).

28. Turgul, V.; Kale, I. On the accuracy of complex permittivity model of glucose/water solutions for non-invasive microwave blood glucose sensing. In Proceedings of the 2015 E-Health and Bioengineering Conference (EHB), Iasi, Romania, 19-21 November 2015; pp. 1-4. [CrossRef]

29. Caduff, A.; Ben Ishai, P.; Feldman, Y. Continuous noninvasive glucose monitoring; water as a relevant marker of glucose uptake in vivo. Biophys. Rev. 2019, 11, 1017-1035. [CrossRef]

30. Karacolak, T.; Moreland, E.C.; Topsakal, E. Cole-cole model for glucose-dependent dielectric properties of blood plasma for continuous glucose monitoring. Microw. Opt. Technol. Lett. 2013, 55, 1160-1164. [CrossRef]

31. Friedrich-Alexander-Universität. Available online: https://www.ethikkommission.fau.de/ (accessed on 20 January 2022). 
32. Elton, D.C. The origin of the Debye relaxation in liquid water and fitting the high frequency excess response. Phys. Chem. Chem. Phys. 2017, 19, 18739-18749. [CrossRef] [PubMed]

33. Rothwell, E.J. Plane-Wave Impulse Response of a Debye Half Space. Electromagnetics 2007, 27, 195-206. [CrossRef]

34. Sewiolo, B. Ultra-Wideband Transmitter Based on M-Sequence for High Resolution Radar and Sensing Application. Doctoral Thesis, Friedrich Alexander Universität, Erlangen, Germany, 2010.

35. Mongraw-Chaffin, M.; Beavers, D.P.; McClain, D.A. Hypoglycemic symptoms in the absence of diabetes: Pilot evidence of clinical hypoglycemia in young women. J. Clin. Transl. Endocrinol. 2019, 18, 100202. [CrossRef] [PubMed] 